\title{
InFLUERCIA DE LOS ACTORES InTERNACIONALES กO-ESTATALES EN LAS ESTRATEGIAS DIPLOMÁTICAS: CONSIDERACIONES DESDE LA COMUNICACIÓn PÚBLICA
}

The influence of the international non-state on the strategies of diplomacy: an approach from public communication

Teresa La Porte Fernández-Alfaro

Universidad de Navarra

E-mail:mtalfaro@unav.es

La proliferación de actores no estatales está transformando gradualmente las relaciones diplomáticas: desafían aquellas establecidas sobre nociones de soberanía a favor de configuraciones alternativas construidas alrededor de valores compartidos o asuntos transnacionales. Los actores no-estatales ejercen un poder de influencia que aconseja la adaptación de la diplomacia tradicional para establecer una relación apropiada con ellos. Aunque la legitimidad de los actores no-estatales para intervenir en diplomacia es controvertida, la hipótesis del trabajo defiende que su legitimidad se basa en la eficacia con que contribuyen a hacer frente a amenazas globales y en la representación cuantitativa o cualitativa de la ciudadanía. Ambas condiciones exigen la habilidad del actor no-estatal para gestionar su comunicación pública. La habilidad comunicativa consiste en difundir mensajes convincentes, de forma coherente con su identidad y capaces de atraer el apoyo del público. El análisis sugiere propuestas para guiar un cambio en la diplomacia que permita aprovechar la relación con estos nuevos actores.

Actores no-estatales; diplomacia; diplomacia pública; comunicación pública; comunicación estratégica 
The proliferation of non-state actors is gradually transforming diplomacy relations: they challenge traditional sovereignty-based notions in favour of alternative configurations built around shared values and transnational issues. Non-state actors exert a power of influence that advices adaptation of traditional diplomacy in order to properly deal with them. Although their legitimacy to develop diplomatic activity is controversial, the hypothesis expressed in the present work assumes that nonstate actors' legitimacy to intervene in international politics lies on their effectiveness in contributing to address global threats and on their representativeness by expressing citizenry concerns. Both, effectiveness and representativeness, depends largely on non-state ability to manage public communication. The communicational ability consists in disseminating a convincing discourse, in communicating coherently with their identity, and in shaping narratives to get public support. The analysis offers some insights to guide a transformation of diplomacy relations in order to take advantage of synergies by dealing with different players.

\section{Introducción}

La proliferación de actores internacionales no-estatales y, sobre todo, el incremento de su poder de influencia, ha obligado a redefinir la actividad propia de la diplomacia. No es factor exclusivo de esta transformación, pero sí determinante. Los estados tienen una larga experiencia en la negociación con los actores no-estatales o en admitirlos como socios en diferentes proyectos internacionales. Lo que resulta novedoso es que el aumento de la autonomía e independencia de estos actores ha desplazado al Estado del control de la acción diplomática o le ha obligado a compartirlo.

La mayor parte de los conflictos actuales, por diferente que sea su naturaleza, conciernen por igual a los actores estatales y no estatales. Son formas diferentes de representar a la ciudadanía, que es la realmente afectada, aunque el fundamento legal y político del Estado sea más sólido. Asuntos como la crisis de Ucrania, la ocupación de Siria e Irak por el DAESH, el brote de ébola y la enfermedad transmitida por el zika, o las consecuencia del tráfico de droga en América Central, implican tanto a los estados como a la Cámara de Comercio Alemana que controla el bloqueo a Rusia, a los líderes religiosos de Irak y Siria que pueden mediar para dificultar el reclutamiento de los integristas, a Médicos sin Fronteras, a los laboratorios farmacéuticos o a la Comisión Interamericana para el Control del Abusos de Drogas.

A pesar de estas circunstancias, la posibilidad de considerar a las organizaciones «no-estatales» como agentes diplomáticos resulta todavía controvertida. Desde el punto de vista jurídico, el Estado es el agente diplomático por excelencia (Convención de Viena, 1964). Sin embargo, ese derecho que el Estado venía ejerciendo de forma absoluta, está ahora condicionado por la influencia de las organización internacionales no-estatales. Desde todas las instancias se recomienda a los gobiernos abrir procesos de diálogo y participación que permitan una interacción con representantes del sector privado, de las ONG, de las confesiones religiosas o de los centros de investigación (Hocking et al., 2012; Golan et al., 2014). Y esto no solo como concesión a una sociedad que exige un mayor protagonismo en la toma de decisiones, sino como condición de eficacia para que los gobiernos logren los objetivos de política exterior que se proponen.

La diplomacia es eminentemente comunicación (Jönsson \& Hall, 2003; Gregory, 2014) y la comunicación la principal actividad desarrollada por la actores no-estatales para ejercitar su poder 
de influencia. El presente trabajo ofrece algunas consideraciones desde la comunicación pública que pueden contribuir a la reflexión sobre el cambio de la estrategia diplomática estatal. Tras una breve descripción del contexto internacional que afecta a actores estatales y no-estatales, el artículo ofrece una síntesis de las diferentes definiciones y clasificaciones de los actores noestatales que mayor incidencia han tenido en el ámbito diplomático. A continuación analiza sus acciones y pondera las circunstancias en que ejercen el poder de influencia que les caracteriza. La conclusión sugiere algunas líneas de adaptación que la diplomacia tradicional debería seguir para favorecer una colaboración eficaz con las organizaciones no estatales.

\section{Contexto internacional}

La emergencia de los actores no-estatales no es el único cambio que afecta al entorno internacional. La generalización del uso de las redes sociales, el protagonismo adquirido por la sociedad civil -tanto en asuntos internacionales como en asuntos nacionales con proyección internacional- y los movimientos masivos de población han contribuido a difuminar las fronteras físicas y virtuales de las naciones y a establecer cauces de relación y decisión que sobrepasan las estructuras de gobierno tradicional. Ese contexto ha provocado a los estados una crisis de legitimidad, eficacia e identidad (Castells, 2008) que, sin perder la autoridad que todavía les compete, les obliga a cambiar la forma de negociar y establecer relaciones con otros actores (Kerr \& Wiseman, 2013).

El propio concepto de poder ha evolucionado desde un concepto más impositivo (hard power) hacia una actitud más igualitaria que requiere el diálogo y la aceptación voluntaria de las políticas (por convencimiento) por parte de los demás actores (soft power: Nye, 2004) o el establecimiento de un equilibrio entre las dos actitudes (smart power: Willson, 2008). Otros análisis proponen una transformación todavía más innovadora y proclaman el establecimiento del «poder social» que consideran menos lineal, más fluido y en el que las relaciones comunicativas juegan un papel esencial (social power: Peter van Ham, 2010). En ese contexto, Naim considera que «el noble arte de gobernar hoy depende de una habilidad mucho más manipulativa y pragmática: la de formar y mantener una coalición» (2013, p. 138).

La diplomacia ejercida por los estados hasta el momento no ha eludido la relación con los actores no-estatales: los gobiernos han sido muy conscientes de la colaboración que las organizaciones noestatales puede aportar desde el punto de vista del conocimiento sobre el terreno, de la experiencia y del respaldo de la sociedad. Pero con frecuencia se han limitado a utilizarlas como fuentes de información o como instrumentos en la aplicación de las medidas decididas sin su presencia. El cambio que la diplomacia estatal debe afrontar ahora es la introducción de esos representantes de la sociedad civil en los procesos de decisión y en la participación efectiva (Hocking et al., 2012).

La relación con los actores no-estatales recae en buena medida sobre la competencia específica de la diplomacia pública. Entendida como la comunicación de los objetivos de política exterior a las audiencias internacionales, le corresponden las acciones de información y establecimiento de relaciones con los públicos. La llamada «nueva diplomacia pública» (Melissen, 2005; Snow \& Taylor, 2009) pone el acento en el establecimiento de relaciones de diálogo con la ciudadanía y de interactuación con las organizaciones que la representan. En lo que a las relaciones se refiere, la diplomacia pública actual está significativamente influida por la «Teoría Relacional» procedente de las relaciones públicas (Lendingham, 2003). Esta teoría enfatiza la identifica- 
ción de criterios de calidad que aseguren que la relación entre la organización (diplomática) y los públicos (actores no-estatales y ciudadanía) es efectiva: es decir, que existe mutualidad en la definición de los objetivos, co-decisión en el diseño de la política y co-participación en su aplicación (Fitzpatrick, 2012; Zaharna, Arsenault \& Fisher, 2013).

\section{Definición de los "actores no-estatales"}

La producción científica sobre el concepto de «actor no-estatal» es muy diversa y fragmentada (Arts et al., 2011). Los estudios más frecuentes se centran en el análisis de casos protagonizados por este tipo de actores, proponiendo definiciones basadas en su comportamiento en lugar de en su naturaleza (Florini, 2000; Higgot et al., 2000).

Desde el punto de vista académico, los análisis más elaborados proceden del constructivismo, hecho que resulta coherente con la importancia que la escuela concede al «constructo social» y al interés que despierta en sus partidarios las iniciativas que surgen de la sociedad civil. De entre todas las propuestas, y a falta de desarrollar un estudio más detenido, parece interesante destacar la definición facilitada por Arts (Arts et al., 2001). Aunque de forma descriptiva y simple, este autor afirma que son actores que ni representan ni han sido creados por los estados, pero operan en el escenario internacional con capacidad potencial de influir en las relaciones internacionales. El mismo autor considera condiciones imprescindibles para que pertenezcan a esta categoría el hecho de que representen a ciudadanos de diversos países, que gocen de reconocimiento de los gobiernos y de los organismos internacionales para intervenir en el ámbito internacional y que actúen dentro de los límites del derecho internacional.

Desde los organismos internacionales, tampoco se aborda con precisión la definición de actor no-estatal. Una de las más precisas procede de la Administración americana: «Entidades no soberanas que ejercitan un significativo poder económico, político, o social y que influyen tanto en un nivel nacional como internacional» (National Intelligence Office, 2007, p. 2). Naciones Unidas no utiliza un concepto único y recurre a la descripción más que a la definición de los organismos involucrados en el asunto que en cada caso interese: seguridad, desarrollo, defensa de los derechos humanos, etc.

La Unión Europea solo propone una definición en el ámbito de la política de desarrollo:

El término de ANE sirve para describir una categoría de organizaciones que reúnen las principales estructuras de la sociedad, existentes o en vías de creación, que no pertenecen al Gobierno ni a la Administración pública. Los ANE son una figura creada voluntariamente por los ciudadanos con el objetivo de promover un debate o defender intereses, generales o específicos. Son independientes del Estado y pueden adoptar la forma de organizaciones con fines lucrativos o no lucrativos. [...] Los ANE asumen funciones de ejecución o de mediación.

El documento continúa identificando y especificando esas funciones: «prestan servicios en ámbitos sensibles», «ejecutan proyectos destinados a satisfacer las necesidades básicas de grupos vulnerables», «desempeñan cada vez más un papel de mediadores, es decir, toman parte en procesos de consulta con los donantes externos y en debates políticos», «contribuyen a definir las estrategias de sus propios países, implicándose así activamente en el proceso de desarrollo» (COM, 2002, pp. 598-final). Las acciones descritas permiten concluir que los actores noestatales desarrollan una función relevante e incisiva en la consecución de los objetivos que

Desde el punto de vista académico, los análisis más elaborados proceden del constructivismo, hecho que resulta coherente con la importancia que la escuela concede al "constructo social" 
la UE se propone en materia de desarrollo, así como en la seguridad o en la consolidación de principios democráticos.

Desde mi punto de vista, es necesario precisar que para que una entidad sea reconocida como actor no-estatal debe adquirir un cierto grado de institucionalización (La Porte, 2012). Es decir, contar con representación oficial, sede social, registro civil, estructura, voluntad de estabilidad y duración en el tiempo, estatutos que recojan su misión, naturaleza y fines. Es importante establecer un mínimo de condiciones que permitan diferenciar a los actores no-estatales de los meros movimientos sociales o iniciativas de activistas aunque sea significativo el impacto social que temporalmente causen.

Por otra parte, es preciso mantener una visión neutral sobre este tipo de actores. El reto que plantean a los actores estatales y la cercanía con la sociedad civil incitan a una espontánea valoración positiva de su intervención. Sin embargo, siendo efectiva en la mayor parte de las ocasiones y reflejando una indudable democratización de la acción internacional, hay aspectos en los que incurren con cierta frecuencia que muestran una debilidad importante: falta de transparencia en la gestión de su financiación, procesos de toma de decisiones confusos, ausencia de evaluación y rendición de cuentas entre otros.

\section{Tipología}

En relación con la tipología de actores no-estatales, podemos distinguir tres criterios básicos de clasificación:

\section{El reto que \\ plantean a los actores estatales \\ y la cercanía con la sociedad civil incitan a una espontánea valoración positiva de su intervención}

- de acuerdo con el derecho penal internacional: legales / ilegales;

- de acuerdo con la procedencia de su financiación: privados / públicos; y

- por los beneficios que reportan sus acciones: empresas o entidades con ánimo de lucro (private sector corporate actors), que a su vez pueden ser transnacionales o multinacionales / o entidades sin ánimo de lucro (ONG), que a su vez se subdividen en las que reciben fondos del estado y las que se financian con fondos privados (Higgott et al., 2000, p. 1).

Esta primera clasificación permite una distinción básica entre estos actores: aquellos que contemplan el desarrollo de actividades ilícitas, como la violencia o la extorsión; aquellos que se mantienen dentro de la legalidad y que dan prioridad a la negociación y al diálogo. Desde el inicio de este trabajo me estoy refiriendo exclusivamente a estos segundos.

Otros autores proceden de forma inductiva y sintetizan la tipología presente en la producción científica (Arts, 2003): organizaciones intergubernamentales, organizaciones no-gubernamentales; organizaciones transnacionales; comunidades epistémicas y una última categoría que agruparía a todas aquellas organizaciones que no entraran en las cuatro categorías anteriores.

Un último sistema de clasificación se basaría en los objetivos y funciones prioritarias de cada organización:

- ONG u organizaciones sociales sin ánimo de lucro (asociaciones, fundaciones);

- organizaciones profesionales sin ánimo de lucro;

- empresas multi/transnacionales;

- grupos y confesiones religiosas; 
- sindicatos, organizaciones políticas, etc., transnacionales;

- comunidades epistémicas: influyen con su investigación y conocimiento (think tanks; centros o grupos de investigación; redes de expertos); y

- grupos de interés: Human Rights Watch, Amnisty International, Greenpeace.

En cualquier caso, todo intento de sistematización de la realidad social es insuficiente o provoca controversia. Por ejemplo, resulta polémica la adscripción de grupos como Amnistía Internacional o Human Rights Watch en la categoría de ONG y hay quienes prefieren considerarlos grupos de interés (advocacy groups, en inglés). También resulta dudosa la clasificación de iniciativas como Wikileaks, dependiendo de la aprobación que se conceda a sus actuaciones.

La afirmación que parece consolidarse es la de excluir a los organismos internacionales de la categoría de actores no-estatales, puesto que, incluso en sus agencias especializadas (en el caso de la ONU) las decisiones dependen de los gobiernos (Art, 2003). Tampoco se incluirían en esta categoría los gobiernos locales o sub-estatales (regionales o municipales): aunque desarrollen una acción exterior independiente de la de los gobiernos nacionales, son órganos electos que actúan (al menos en teoría) de forma coordinada con la política exterior oficial. La única excepción se haría con aquellas instituciones de la Unión Europea que realmente sean transnacionales y no inter-gubernamentales (Comisión, Parlamento Europeo).

\section{Acciones que desempeñan}

De acuerdo con la producción científica que ha abordado el análisis de los actores no estatales de forma genérica, puede distinguirse tres ámbitos de actuación en los que intervienen con acciones específicas (Higgott et al., 2000; Arts et al., 2011).

1. En su relación con los actores estatales y con otros actores no-estatales (por iniciativa propia o respondiendo a una solicitud de colaboración). Las acciones clásicas son advertir, denunciar, proponer, presionar y colaborar. Los actos específicos serían:

- configurar la agenda política, económica y social de los actores estatales u organismos intergubernamentales provocando que figuren en los asuntos tratados sus intereses y que sean considerados como prioridades;

- aportar información sobre las causas, entidad y alcance de problemas sociales, políticos y económicos;

- acercar la opinión, necesidades e intereses del ciudadano al ámbito institucional;

- facilitar conocimiento especializado en los procesos de toma de decisión para garantizar medidas adecuadas;

- asesorar sobre la información y aplicación de esas medidas; y

- colaborar en la puesta en práctica de políticas decididas en entidades estatales.

2. En su relación con la opinión pública las acciones tradicionales serían informar, denunciar, alarmar y solicitar respaldo (estable: voluntarios, donantes / ocasional: respaldo eventual de protestas). En el ámbito internacional, podrían distinguirse dos públicos diferentes:

- público del entorno en que surge el actor no-estatal: con una cultura política similar y en situación de prestar colaboración; y 
- público del entorno en el que el actor no-estatal actúa: con una política cultura diferente y en situación de recibir colaboración.

3. En su relación con los socios, afiliados, miembros, etc. las acciones que desarrollan los actores no-estatales son: información institucional, fomento de la participación en foros propios y canalización de la contribución de los miembros a los procesos de decisión interna.

Los actores políticos no-estatales pueden actuar en colaboración con los estados, con organismos internacionales gubernamentales, con otros actores no-estales confines similares o complementarios, o, por el contrario, emprender acciones en solitario. La independencia con que actúen en cada una de esas iniciativas dependerá de las condiciones de financiación y de la delimitación del área de intervención que se haya estipulado en el acuerdo original.

En un intento de articular algunas de las afirmaciones realizadas hasta el momento, el siguiente cuadro podría sintetizar las acciones más características de estos actores, de acuerdo con su ámbito de acción y con el nivel en que pretendan ejercer su influencia.

\section{Tabla 1. Acciones de los actores}

\begin{tabular}{|c|c|c|}
\hline Influencia & Ámbito & Acciones \\
\hline En la toma de decisiones. & $\begin{array}{l}\text { Instituciones internacionales. } \\
\text { Otros agentes no-estatales. } \\
\text { Gobiernos/organismos locales. }\end{array}$ & $\begin{array}{l}\text { Información sobre intereses propios y análisis de la } \\
\text { situación. } \\
\text { Participación en debates. } \\
\text { Participación en procesos de decisión. } \\
\text { Presión o lobby. } \\
\text { Creación de coaliciones. }\end{array}$ \\
\hline En los discursos públicos. & $\begin{array}{l}\text { Políticos e institucionales. Medios } \\
\text { tradicionales. } \\
\text { Redes sociales. }\end{array}$ & $\begin{array}{l}\text { Captar la atención. } \\
\text { Sensibilizar. } \\
\text { Interpretación propia de asuntos públicos (framing). }\end{array}$ \\
\hline En las regulaciones. & $\begin{array}{l}\text { Instituciones europeas. } \\
\text { Gobiernos nacionales/locales. }\end{array}$ & $\begin{array}{l}\text { Denunciando disfunciones. } \\
\text { Recabando apoyo expertos. } \\
\text { Participando en reformas, con: conocimiento y } \\
\text { experiencia, conocimiento de la soc. civil, } \\
\text { intereses propios. }\end{array}$ \\
\hline En la acción. & $\begin{array}{l}\text { Áreas de acción internacionales de los } \\
\text { actores no-estatales: } \\
\text { zonas geográficas con riesgos } \\
\text { medioambientales, } \\
\text { áreas de cooperación, } \\
\text { mercados propios. }\end{array}$ & $\begin{array}{l}\text { Continuar procesos de co-decisión y seguimiento. } \\
\text { Procesos de evaluación. } \\
\text { Cultivo del apoyo (donantes, voluntarios, soc. civil...). } \\
\text { Negociación con agentes implicados. } \\
\text { Desarrollo de los proyectos. }\end{array}$ \\
\hline
\end{tabular}

Fuente: Elaboración propia

\section{Ejercicio del poder de influencia}

El poder ${ }^{1}$ de los actores no-estatales es poder de influencia ${ }^{2}$. Es decir, se fundamenta en su habilidad para configurar las decisiones de los que, por el momento, conservan la capacidad de legislar, juzgar y ejecutar en el espacio internacional: los gobiernos nacionales (o actores estata-

\section{Los actores} no-estatales pueden actuar en colaboración con los estados, con organismos internacionales, con otros actores no-estales o, por el contrario, emprender acciones en solitario

1 De acuerdo con Joseph Nye, el poder en el ámbito social es «la capacidad de influir en otros para conseguir los resultados que uno desea» $(2011$, p. 6).

2 Aunque la discusión sobre este concepto y sobre la relación misma entre «influencia» y «poder» es muy extensa, en este trabajo se entiende que el poder de influencia es la capacidad de cambiar el comportamiento de alguien/ algo modificando su forma de entender o percibir una situación (Zimmerling, 2005, p. 146). 
les). También es denominado «micro poder»: poder de vetar, contrarrestar, combatir y limitar el margen de maniobra de los grandes actores (Naim, 2013).

Una cuestión previa a la descripción del tipo de poder que ejercen es la autoridad con que lo hace. ¿En qué se basa o qué lo determina? El poder de influencia de los actores no estatales está determinado por cómo se perciba públicamente su legitimidad ${ }^{3}$ para intervenir en unas circunstancias determinadas. Esa legitimidad se fundamenta en la representatividad ${ }^{4}$ que demuestren tener, tanto en términos cuantitativos (población civil que les respalda) como cualitativos (expertos o autoridades políticas que les avalen) y en la eficacia ${ }^{5}$ con que sean capaces de afrontar o resolver un problema, amenaza o conflicto internacional (Avant, Finnemore \& Sell, 2010; Heard-Laureote, 2010; La Porte, 2012).

La representatividad demostraría que la organización está suficientemente respaldada por la ciudadanía, bien por la cantidad de ciudadanos que comparten sus objetivos o bien por la cualificación de aquellos la apoyan. En cierta medida, esta representatividad suplantaría la legitimidad democrática que los estados reciben en las urnas. Evidentemente, esta afirmación suscita controversia, porque el apoyo es volátil, no comprometido y resulta difícil valorar el soporte real en un momento determinado. El segundo pilar que justifica la legitimidad de los actores no estatales es la eficacia: es decir, la capacidad que tienen para resolver los problemas que les competen. Amnistía Internacional reduciendo encarcelamientos o penas de muerte arbitrarias, líderes musulmanes moderando el discurso radical, u ONG de ayuda humanitaria ayudando a la atención de los refugiados sirios, ofrecen un conocimiento especializado y una acción en el origen del conflicto con la que, no solo suple al Estado, sino que le orienta y dirige en la adopción de medidas posteriores, garantizando su adecuación y eficacia.

Los límites a ese poder resultan obvios. Por el momento, no legislan (aunque que su capacidad normativa es cada vez mayor (Gardner, 2010) y carecen de poder económico, militar o político. Como se afirmaba al principio, su poder se limita a la influencia que pueden ejercer en quienes tienen la capacidad de legislar o de ejecutar (Zimmerling, 2005).

El ejercicio del poder de influencia se canaliza principalmente a través de las acciones de comunicación pública. La comunicación es esencial para el ejercicio de la influencia de los actores no estatales porque es condicionante para:

- dar a conocer su existencia y sus objetivos (Van Rooy, 1997);

- colaborar, dialogar o involucrar a otros actores sociales en la consecución de sus propósitos (Holzschiter, 2005; Castells, 2009); y

- construir una imagen pública que les legitime como actores políticos y sociales, construida a partir de su eficacia y de su representatividad (Higgot et al., 2000; Bernstein \& Cashore, 2007).

3 El concepto de «legitimidad» adoptada en este trabajo sigue la definición propuesta por Edwards (1999, p. 26) y que entiende que es «el derecho de ser o de hacer algo en sociedad». Refleja que una organización está constituida conforme al derecho, tiene fines apropiados, admisibles y está justificada para hacer lo que hace y decir lo que dice, contando con el apoyo de un sector de la población identificable.

4 De acuerdo con Jönsson (2008) parece crucial para la legitimar la actuación de un actor no-estatal exigir información que demuestre el apoyo ciudadano, ya que no cuenta con el refrendo de las urnas.

5 Habría diversos criterios para evaluar la eficacia de actor no-estatal. El más objetivo sería el que él mismo establezca, de acuerdo con el propósito que la mueva a desarrollar una acción: captar la atención, denunciar, condenar, presionar, innovar o modificar la legislación, aplicar una política, etc.

\section{El poder de influencia de}

los actores no estatales está determinado por cómo se perciba públicamente su legitimidad 
La influencia es mayor cuando esas acciones comunicativas demuestran algunas destrezas (La Porte, 2015). Desde el punto de vista de la comunicación pública es importante, en primer lugar, que las agendas internacionales de estos actores resulten relevantes, incluyan intereses prioritarios para el conjunto de la comunidad internacional, propongan soluciones innovadoras y, al tiempo, sean lo suficientemente flexibles como para negociar e incluir los intereses de otros actores entre sus objetivos. En este sentido, se puede destacar la colaboración establecida entre actores estatales, ONG y representantes del sector privado (como son el gobierno británico, la Fundación Gates y Médicos sin Fronteras) para adaptar sus agendas e integrar en la misma cadena de acción la financiación, producción y distribución de vacunas contra enfermedades endémicas en zonas de subdesarrollo.

En segundo lugar, la narrativa utilizada tanto para presentar públicamente la institución como para explicar los objetivos de su política, debe reflejar con coherencia la identidad de la organización y exponer una actitud de abierta colaboración. Estos rasgos aumentarán su credibilidad, puesto que se da a conocer con transparencia, reflejando los principios que le animan, fortalezas y también limitaciones: debe haber una concordancia entre los que el actor no-estatal dice que es, lo que hace y lo se propone hacer. El discurso utilizado es una de las principales estrategias con que los actores no-estatales cuentan para influir: la visibilidad que den a ciertos aspectos, la manera de interpretar o enfocar un asunto, la calidad de su expresión y la habilidad para invitar a la acción de forma atractiva son elementos esenciales para causar impacto, movilizar partidarios y conseguir respaldos duraderos. En algunas ocasiones, los actores no-estatales son los primeros en abordar un conflicto y su primera explicación determinará la forma en que este sea definido y entendido posteriormente por la opinión pública y por los estados. Hay muchos ejemplos que podrían incluirse aquí: baste ahora con citar la pericia de las ONG de defensa de derechos de la mujer para unir esos asuntos a las cuestiones de desarrollo, o reenfocar la amenaza del virus VHS desde una cuestión meramente científica a cuestión humana resaltando su faceta de enfermedad (Holzscheiter, 2005).

En tercer lugar, es importante destacar que la «narrativa» se construye también con las acciones. La narrativa no es exclusivamente un mensaje oral o escrito: incluye la actitud y el comportamiento de los que difunden o defienden el mensaje y está intrínsecamente unido al objetivo que se pretenda lograr. En este sentido, las instituciones que intenta promover los valores democráticos (universidades americanas en El Cairo o en Dubai) deben demostrar en sus relaciones lo que destacan en su discurso y valorar el diálogo real y su disposición a co-participar con los públicos. En relación con los objetivos, la agresividad de algunas de las acciones de Greenpeace son justificables en la medida en que su propósito es captar la atención de públicos y agentes reguladores; para conseguir un cambio de regulación duradero, otros actores que también defienden el medio ambiente, prefieren hacerlo a través del rigor de informes y estudios científicos que son presentados en los centros de poder (World Resources Institute).

Para terminar, es importante destacar el tipo de relaciones que los actores no-estatales estén dispuestos a establecer, tanto con los estados como otras organizaciones de la sociedad civil. Como el nuevo entorno global aconseja también a los estados, estas deben permitir la integración de las diferentes fortalezas de los interlocutores, favorecer que exista co-creación en el diseño de las políticas, de las acciones conjuntas o de las soluciones y que exista colaboración en el momento de desarrollar esa actividad conjunta (Hudson \& Ansted, 2008).

\section{En algunas ocasiones, los actores no-estatales son los primeros en abordar un conflicto y su primera explicación determinará la forma en que este sea definido $y$ entendido posteriormente}




\section{Conclusiones: posibles líneas de cambio}

El actual contexto internacional ha sufrido una rápida transformación en las dos últimas décadas. Destacando solo aquellos cambios que afectan al ejercicio del poder se pueden señalar dos aspectos esenciales: su difusión entre diversos agentes y la necesidad de establecer coaliciones para ejercerlo con eficacia (Naim, 2013). En el ámbito de la diplomacia, Wiseman explica que esta situación ha llevado a un «polilateralismo» en el que diversos actores de naturaleza distinta deben actuar en colaboración, independientemente de su legitimidad para ejercer funciones diplomáticas (Wiseman, 2004).

Aunque la diplomacia permanezca siendo una competencia exclusiva del Estado, ya no puede desarrollarla de forma tan independiente o autónoma como venía haciendo y su ejercicio requiere una mayor capacidad de persuasión para convencer a sus interlocutores en lugar de la simple imposición de normas y criterios.

Algunas sugerencias para que las prácticas tradicionales de diplomacia desarrolladas por el Estado se adapten a la colaboración con actores no-estatales y aprovechen las sinergias que la acción conjunta reporta, podrían ser las siguientes:

- Aunque la agenda atienda prioritariamente a los objetivos de la política exterior del gobierno, debe ser lo suficientemente abierta como para dar cabida a la colaboración con actores que tengan objetivos similares. La mentalidad con que se elabore debe buscar la complementariedad no la exclusividad o la competencia.

- La actitud de los diplomáticos debe mostrar capacidad para trabajar con otros y facilitar la colaboración y participación de actores ajenos al Estado.

- Las relaciones deben ser flexibles y tener capacidad de improvisación: aunque se mantenga la distinción entre relaciones bilaterales y multilaterales, muchos de los contactos se producirán de manera informal en escenarios improvisados (Hocking et el., 2012).

- La acción diplomática se desarrollará de forma menos independiente: el Estado tiene que conocer qué actores no-estatales actúan en el área de interés y valorar la oportunidad de trabajar en colaboración con ellos, participando en redes que se hayan establecido antes de su llegada (Hocking et el., 2012).

- La diplomacia pública ha incrementado su importancia y debe intervenir en el propio diseño de la agenda para presentarla de forma atractiva e influyente. A la diplomacia pública le corresponde también elaborar una narrativa capaz de estar presente en el debate internacional generado por otros, aportando a la interpretaciones innovadoras que le permitan liderar en aquellas áreas que el Estado considere cruciales.

\section{Bibliografía}

Arts, B. (2003). Non-State Actors in Global Governance. A Power Analysis. Artículo presentado en ECPR Joint Sessions. Edimburgo, Escocia.

Arts, B., Noortmann, M. \& Reinalda, B. (2001). Non-state actors in international relations. Aldershot: Ashgate.

Avant, D., Finnemore, M. \& Sell, S. (2010). Conclusion: authority, legitimacy, and accountability in global politics. En D. Avant, M. Finnemore \& S. Sell (Eds.), Who Governs the Globe? Cambridge: Cambridge University Press.
Aunque la diplomacia permanezca siendo una competencia exclusiva del Estado, ya no puede desarrollarla de forma tan independiente 0 autónoma como venía haciendo 
Bernstein, S. \& Cashore, B. (2007). Can Non-State Global Governance Be Legitimate? An Analytical Framework. Regulation and Governance, 1, 347-371.

Castells, M. (2008). The New Public Sphere: Global Civil Socety, Communication Networks, and Global Governance. The ANNALS of the American Academy of Political and Social Science, 616(1), 78-93.

Castells, M. (2009). Comunicación y poder. Barcelona: Alianza Editorial.

Comisión de las Comunidades Europeas. (2002, 07 de noviembre). Participación de los actores no estatales en la política comunitaria de desarrollo. Comunicación de la Comisión al Consejo, al Parlamento y al Comité Económico y Social. Bruselas.

Edwards, M. (1999). International Development NGOs: Agents of Foreign Aid or Vehicles of International Cooperation? Non-Profit and Voluntary Sector Quarterly, 28, 25-37.

Fitzpatrick, K. (2012). Defining Strategic Publics in a Networked World: Public Diplomacy's challenge at home and abroad. The Hague Journal of Public Diplomacy, 7, 421-440.

Florini, A. M. (Ed.). (2000). The Third Force: The Rise of Transnational Civil Society. Washington D. C.: Carnegie Endowment for International Peace.

Gardner, A. M. (2010). Democratic Governance and Non-State Actors. Nueva York: Palgrave Macmillan.

Golan, G. J., Yang, S. U. \& Kinsey, D. (2014). International Public Relations and Public Diplomacy: Communication and Engagement. Nueva York: Peter Lang.

Gregory, B. (2014). The Paradox of US Public Diplomacy: Its Rise and "Demise". Institute for Public Diplomacy and Global Communication. Washington, D. C.: George Washington University.

Heard-Laureote, K. (2010). European Union Governance. Effectiveness and Legitimacy of European Commission Committees. Londres \& Nueva York: Routledge.

Higgott, R., Underhill, G. \& Bieler, A. (Eds.). (2000). Non-state Actors and Authority in the Global System. Londres: Rutledge.

Hocking, B., Melissen, J., Riordan, S. \& Sharp, P. (2013). The Future of Diplomacy. La Haya: Clingendael Neatherlands Institute of International Relations.

Holzschiter, A. (2005). Discourse as Capability: Non-State Actors' Capital in Global Governance. Millenium-Journal of International Studies, 33, 723-746.

Hudson, L. \& Ansted A. (2008). How Government, Business, and Non-Governmental Organizations Can Work Together to Address Global Challenges. En J. Welsh \& D. Fera (Eds.), Engagement. Public Diplomacy in a Globalized World (pp. 147-159). Londres: Foreign Office \& Commonwealth Office.

Jönsson, C. (2008). Global Governance: Challenges to Diplomatic Communication Representation and Recognition. En A. Cooper, B. Hocking \& W. Maley (Eds.), Global Governance and Diplomacy: Worlds Apart? (pp. 29-39). Londres: Palgrave-MacMillan.

Jönsson, C. \& Hall, M. (2003). Communications: An essential aspect of diplomacy. International Studies Perspectives, 4(2), 195-210. 
Kerr, P. \& Wiseman, G. (2013). Diplomacy in a Globalizing World. Theories and Practices. Oxford: Oxford University Press.

La Porte, T. (2012). The Impact of 'Intermestic' Non-State Actors on the Conceptual Framework of Public Diplomacy. The Hague Journal of Diplomacy, 7, 445-463.

La Porte, T. (2015). Improving Efficiency in Public Diplomacy Practices: Advising Non-state actors Strategies in the EU framework. En M. L. Azpiroz (Ed.), EU and Latin America public diplomacy: new trends and proposals. Bruselas: Peter Lang.

Ledingham, J. A. (2003). Explicating relationship management as a general theory of public relations. Journal of Public Relations Research, 15(2), 181-198.

Melissen, J. (Ed.). (2005). The New Public Diplomacy. Nueva York: Palgrave Macmillan.

Naím, M. (2013). El fin del poder. Barcelona: Debate.

Nye, J. (2004). Soft Power. Nueva York: Public Affairs.

Nye, J. (2011). The Future of Power. Nueva York: Public Affairs.

Snow, N. \& Taylor, P. (Eds.). (2009). Routledge Handbook of Public Diplomacy. Londres: Routledge.

US National Intelligence Council. (2007). Nonstate Actors: Impact on International Relations and Implications for the United States. National Intelligence Council. Washington, D. C. Recuperado de http://www.fas.org/irp/nic/nonstate_actors_2007.pdf

Van Ham, P. (2010). Social Power in International Politics. Londres: Routledge.

Van Rooy, A. (1997). A New Diplomacy? How Ambassadors (Should) Deal with Civil Society Organizations. En R. Wolfe (Ed.), Diplomatic Missions: The Ambassador in the Canadian Foreign Policy. Kingston: Queen's University.

Willson, E. J. (2008). Hard Power, Soft Power, Smart Power. The ANNALS of the American Academy of Political and Social Science, 616(1), 110-124.

Wiseman, G. (2004). "Polylateralism" and New Modes of Global Dialogue. En C. Jönsson \& R. Langhorne (Eds.), Diplomacy, vol. III (pp. 36-57). Londres: Sage.

Zaharna, R. S., Arsenault, A. \& Fisher, A. (2013). Relational, networked and collaborative approaches to public diplomacy. Nueva York: Routledge.

Zimmerling, R. (2005). Power and Influence. Dordrecht, Holanda: Springer. 